\title{
Designing a One-Dimensional Photonic Crystal via Thue-Morse Sequence Containing Two Types of Double Negative Metamaterial
}

\section{Ali Baseri}

Shiraz University of Technology

Alireza Keshavarz ( $\nabla$ keshavarz@sutech.ac.ir)

Shiraz University of Technology https://orcid.org/0000-0003-3637-7005

\section{Research Article}

Keywords: Photonic crystal, Quasi-periodic sequence, Metamaterial, Photonic band-gap, Transfer matrix method

Posted Date: May 10th, 2021

DOI: https://doi.org/10.21203/rs.3.rs-388942/v1

License: (c) (1) This work is licensed under a Creative Commons Attribution 4.0 International License.

Read Full License 


\title{
Designing a one-dimensional photonic crystal via Thue-Morse sequence containing two types of double negative metamaterial
}

\author{
Ali Baseri • Alireza Keshavarz
}

Received: date / Accepted: date

\begin{abstract}
This study investigates the propagating of electromagnetic waves through a one-dimensional quasi-photonic crystal with the transfer matrix method. Our proposed structure consists of two types of double negative metamaterials, organized according to the Thue-Morse sequence law. The results show that changing the structure via quasi-periodic arrangements makes the outcome more varied than applying the absolute periodic arrangement. Given that, our desirable results of interest are more conveniently achieved. The structure completely stops both $\mathrm{s}$ and $\mathrm{p}$ polarization at the lower frequencies, for all incidence angles. It also partially stops s and p polarization, at higher frequencies. Moreover, the achieved transmittance spectrum contains several omnidirectional band-gaps, which remain invariant with changes in the incidence angle. The oscillation of the transmittance values also becomes more intense at higher orders of the period number. This study could pave the way for optimizing of photonic crystal circuits, splitters, switches, etc.
\end{abstract}

Keywords Photonic crystal · Quasi-periodic sequence - Metamaterial · Photonic band-gap · Transfer matrix method

\section{Introduction}

The prediction of metamaterials or negative-index materials (NIMs) by Veselago [1] has established a number of new science and technologies. Due to their various interesting properties such as negative refraction index, antiparallel phase-energy velocities, magnetism in optics and cloaking [2], metamaterials have a number of unique applications such as magnetic resonance imaging (sensing), energy harvesting [3], superlens as well as focusing, collimating and filtering light [4]. The

A. Baseri

Physics Department, Shiraz University of Technology, Shiraz, Iran

E-mail: a.baseri@sutech.ac.ir

A. Keshavarz

Physics Department, Shiraz University of Technology, Shiraz, Iran

E-mail: keshavarz@sutech.ac.ir 
development of metamaterial based sciences paved the way for the progress of the following fields: photonics, material science, nanotechnology, Terahertz technology, thermodynamics, electronics, acoustics, etc.

Photonic crystal (PC) medium consists of two or several constitutive elements, whose difference is characterized based on refraction indices. PCs are usually of great interest due to their photonic band-gaps (PBGs) that stop the transmitted light in some particular frequency regions. Utilizing the unconventional substances such as metamaterial, superconductor, semiconductor [5], plasma [6,7], liquid crystal [8], etc. obviates the drawbacks of conventional PCs.

Recently, the PCs consisting metamaterials have been a focal point due to their beneficial features. PC structures might have several types of arrangement such as absolute periodic, quasi-periodic (QP), and of course another new type of arrangement named mixed-quasi-periodic (MQP) [9]. In this paper, we focus on the QP arrangement, which their constitutive elements are not positioned periodically, and their repetition obeys the following mathematic laws; the Fibonacci sequence, the Thue-Morse (T-M) sequence [10], the double sequence, the fractal [11] as well as the pre-fractal Cantor, the maximum length sequence (MLS), etc.

Merlin et al. [12] used the Fibonacci sequence in QP systems for the first time. These types of media have been utilized in various fields of photonics. $\mathrm{Xu}$ et al. [13] found defect modes in a one-dimensional PC (1DPC) with a double negative (DNG) metamaterial defect layer. Liang et al. [14] investigated a type of PBG as an unusual narrow transmission band in a PC structure, containing right-hand material (RHM) and left-hand material (LHM). Shadrivov et al. [15] proposed a 1DPC structure that utilized a transparent LHM so that it could trap light in a three-dimensional space. Now, the PC structures containing metamaterials are considered as metamaterial PCs, or in simple terms, MetaPCs. The fabulous features of the MetaPCs have been considerably overlooked in the recent decade [16-20]. Liviotti [21] applied a transformed wavelet to a T-M PC, and showed that this technique was successful in estimating the scaling exports and finding the wavevector associated with them. Vasconcelos et al. [22] investigated the transmission quantity of a T-M QP multilayer slab, and compared the different behaviour of the oblique- and normal-incidence transmission spectra over a particular range of frequency. Deng et al. [23] proposed an omnidirectional filter containing singlenegative (SNG) metamaterials, which was tunable with polarization of light, the incidence angle of light, and lattice constants.

The salient difference of the current study with the previous analogous researches is the use of a sixteen-layer of the T-M sequence with two types of DNG metamaterials. Furthermore, the complete and partially (tunable) omnidirectional PBGs are studied in several frequency ranges. In addition, a dielectric-type layer is substituted instead of one type of the metamaterial layers, so that the structure consists of metamaterial and dielectric layers. Finally, the consequences of the substitution under several permittivities of the dielectric are compared with the previous sections.

The concepts, basic equations of the proposed structure as well as the method and required descriptions regarding the simulation are presented in section 2 . The analysis and findings are expanded in section 3. Finally, the conclusion is presented in section 4. 


\section{Model and basic equations}

In this study, the approach of obtaining the transmittance and reflectance quantities is based on the transfer matrix method (TMM). In this method, each layer of the multilayer structure corresponds to its own transfer matrix, so that the matrix (relation (1)) relates the incident beam to the next layer, from the previous layer. If the transfer matrix of a period obtained by multiplying the transfer matrix of each layer in a period, which is considered as $M_{P}$, the total transfer matrix of the structure with $N$ period will be defined as equation (2) [24-27]:

$$
\begin{gathered}
M_{j}\left[d_{j}\right]=\left[\begin{array}{cc}
\cos k_{j z} d_{j} & \frac{-i}{p_{j}} \sin k_{j z} d_{j} \\
-i p_{j} \sin k_{j z} d_{j} & \cos k_{j z} d_{j}
\end{array}\right], \\
{\left[M_{P}\right]^{N}=\left[M_{1} \cdots M_{\ell}\right]^{N}=\left[\begin{array}{ll}
m_{11} & m_{12} \\
m_{21} & m_{22}
\end{array}\right] ; \ell, N \in \mathbb{N} ; 1<j<\ell,}
\end{gathered}
$$

where $k_{j z}=(\omega / c) \sqrt{\varepsilon_{j}} \sqrt{\mu_{j}} \sqrt{1-\left(\sin ^{2} \theta / \varepsilon_{j} \mu_{j}\right)}$ indicates the wavenumber, $d_{j}$ is the thickness of $j^{t h}$ layer, $c$ is the speed of light in vacuum, and the following parameters are associated with s and p polarization, respectively:

$$
\begin{aligned}
& p_{j s}=\left(\sqrt{\varepsilon_{j}} / \sqrt{\mu_{j}}\right) \sqrt{1-\left(\sin ^{2} \theta / \varepsilon_{j} \mu_{j}\right)} \\
& p_{j p}=\left(\sqrt{\mu_{j}} / \sqrt{\varepsilon_{j}}\right) \sqrt{1-\left(\sin ^{2} \theta / \varepsilon_{j} \mu_{j}\right)},
\end{aligned}
$$

where $\varepsilon_{j}$ and $\mu_{j}$ are respectively the permittivity and the permeability of $j^{t h}$ layer, and $\theta$ is the incidence angle of the light from normal axis of the structure. The refraction indices of the metamaterials are defined as $n_{j}= \pm\left[\varepsilon_{j} \mu_{j}\right]^{1 / 2}$. If both $\varepsilon_{j}$ and $\mu_{j}$ are negative, $n_{j}$ will take the sign - . Otherwise, it will take the sign + . Permittivity and permeability of the first $(A)$ and the $(B)$ second metamaterial layers are:

$$
\begin{gathered}
\varepsilon_{A}=1.0+\frac{25}{0.9^{2}-f^{2}}+\frac{100}{11.5^{2}-f^{2}}, \mu_{A}=1.0+\frac{9}{0.902^{2}-f^{2}} \\
\varepsilon_{B}=1.0-\frac{100}{f^{2}}, \mu_{B}=1.44-\frac{100}{f^{2}}
\end{gathered}
$$

where $f$ indicates the frequency in the $\mathrm{GHz}$ range. In the proposed structure, layer $A$ is a DNG metamaterial at the frequency range of $1 \mathrm{GHz}$ to $3.78 \mathrm{GHz}$, in which its both $\mu$ and $\varepsilon$ are negative. However, layer $A$ has positive $\mu$ and negative $\varepsilon$ at the range of $3.5 \mathrm{GHz}<f<3.78 \mathrm{GHz}$, in which this type of substances is called epsilon-negative (ENG) metamaterial. For the frequencies $f>3.78 \mathrm{GHz}$, both $\varepsilon$ and $\mu$ of the layer get a positive sign, and the layer becomes positive index material (PIM). Layer B is DNG metamaterial in the range of $1 \mathrm{GHz}$ to $8.33 \mathrm{GHz}$. It is ENG metamaterial in the range of $8.33 \mathrm{GHz}$ to $10 \mathrm{GHz}$, and it is PIM for $f>10 \mathrm{GHz}$, for both $\mathrm{s}$ and $\mathrm{p}$ polarization of the incident light. Fig. 1 presents more details about $\varepsilon, \mu$ and refraction indices $n$ of the metamaterials. The transmission and reflection coefficients of the structure are defined in accordance with equation (2), as follows:

$$
\begin{gathered}
t(f)=\frac{2 p_{0}}{\left(m_{11}+m_{12} p_{s}\right) p_{0}+\left(m_{21}+m_{22} p_{s}\right)}, \\
r(f)=\frac{\left(m_{11}-m_{22}\right) \cos \theta-\left(m_{12} \cos ^{2} \theta-m_{21}\right)}{\left(m_{11}+m_{22}\right) \cos \theta-\left(m_{12} \cos ^{2} \theta+m_{21}\right)},
\end{gathered}
$$



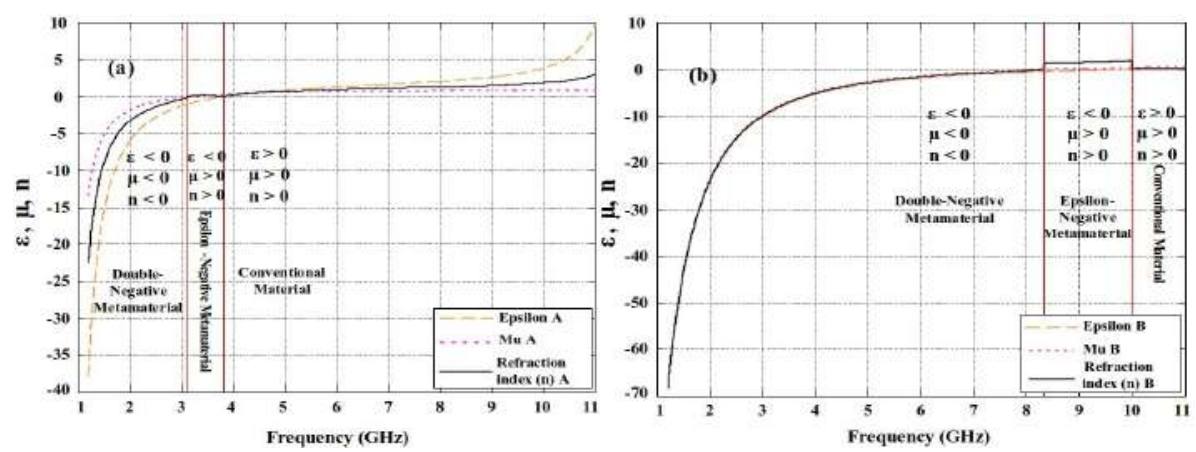

Fig. 1 The function of permittivity, permeability and refraction index of metamaterials (a) A and (b) B in terms of frequency. According to the diagrams, type of the metamaterials changes across different frequency ranges.

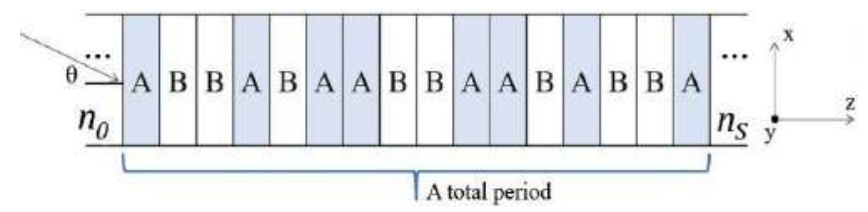

Fig. 2 A schematic for a $1 \mathrm{D}$ quasi-PC of a sixteen-layer T-M sequence, which contains two types of DNG metamaterials as layers A and B with refraction indices $n_{A}$ and $n_{B}$, respectively. $n_{0}$ and $n_{s}$ are considered as the refraction index of air, and $\theta$ is the incidence angle.

where $p_{0}=n_{0} \cos \theta$ and $p_{s}=n_{s} \cos \theta$ are respectively associated with the initial and final medium adjoin the multilayer. Finally, the transmittance and reflectance are respectively given as $T=\left(p_{s} / p_{0}\right)|t(f)|^{2}$ and $R=|r(f)|^{2}$, which they could be applied in the relation $R+T=1$.

In this study, $\mathrm{GHz}$ wave propagation in a sixteen-layer T-M (fourth-order of T-M sequence) quasi-PC structure is simulated in accordance with Fig. 2, under several certain parameters. By applying the following substitution rules: $A \rightarrow A B$ and $B \rightarrow B A$, we can deduce all subsequent orders of T-M sequence as follows: $S_{0}=A, S_{1}=A B, S_{2}=A B B A, S_{3}=A B B A B A A B, S_{4}=A B B A B A A B$ and so on [28].

\section{Tunable quasi-PC filter, results and discussion}

\subsection{Incidence angle}

The transmittance of the proposed structure, which consists of two types of metamaterial layers, is evaluated under several incident angles as $\theta=0^{\circ}, 30^{\circ}, 65^{\circ}$, and $88^{\circ}$ for both $\mathrm{p}$ and $\mathrm{s}$ polarization, and thickness of the layers are $d_{A}=4 \mathrm{~mm}$ and $d_{B}=5 \mathrm{~mm}$. The total period number of the structure is considered as $N=5$. The transmittance spectrum of the structure is demonstrated versus several frequency ranges in Figures 3, 4 and 5. As shown in Fig. 3(TE), the band-gaps negligibly move towards lower frequencies as the incidence angle increases. For example, the 


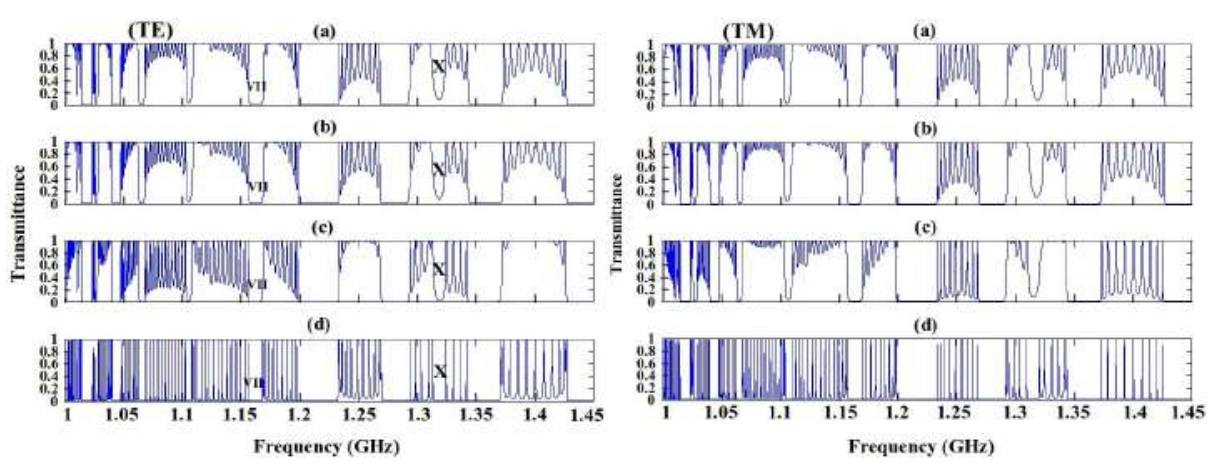

Fig. 3 The transmittance spectrum of the incident wave in the frequency range of $1 \mathrm{GHz}$ to $1.45 \mathrm{GHz}$, to a quasi-PC, which contains two types of DNG metamaterial with the fourth-order of the T-M arrangement for the TE and TM polarization under several incidence angles of (a) $0^{\circ}$, (b) $30^{\circ}$, (c) $65^{\circ}$ and (d) $88^{\circ}$.
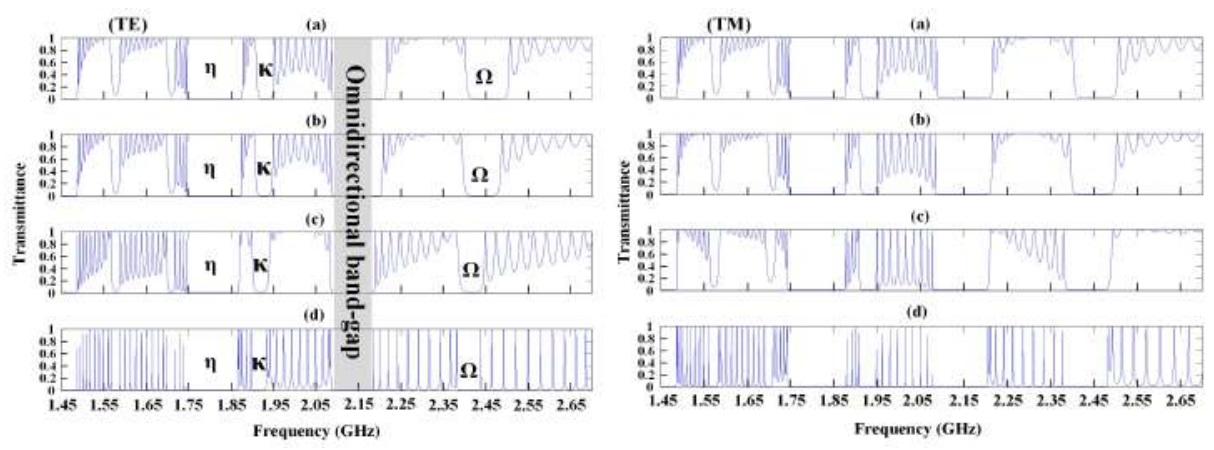

Fig. 4 The transmittance spectrum of the incident wave in the frequency range of $1.45 \mathrm{GHz}$ to $2.7 \mathrm{GHz}$, to a quasi-PC, which contains two types of DNG metamaterial with the fourth-order of the T-M arrangement for the TE and TM polarization under several incidence angles of (a) $0^{\circ}$, (b) $30^{\circ}$, (c) $65^{\circ}$ and (d) $88^{\circ}$.
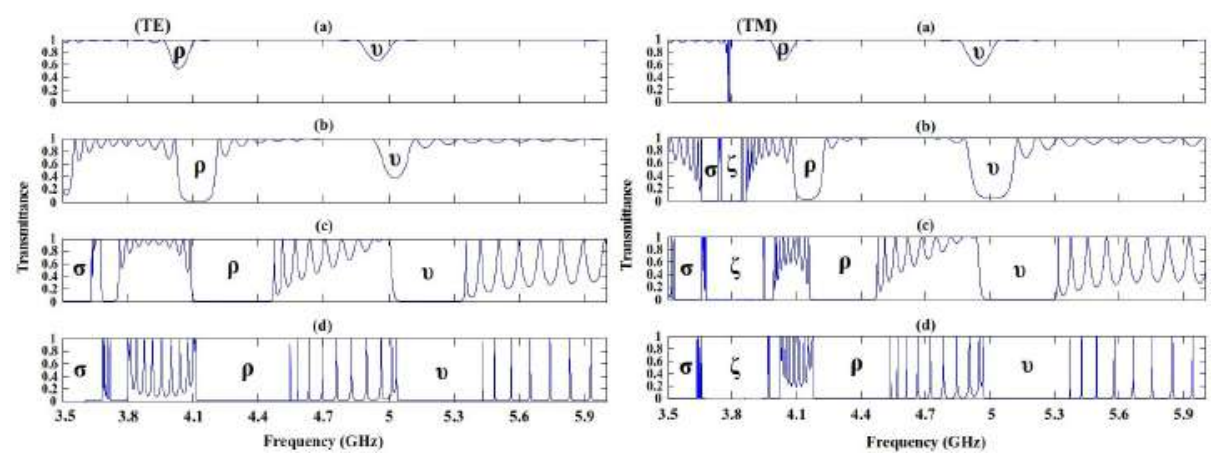

Fig. 5 The transmittance spectrum of the incident wave in the frequency range of $3.5 \mathrm{GHz}$ to 6.0 GHz, to a quasi-PC, which contains two types of DNG metamaterial with the fourth-order of the T-M arrangement for the TE and TM polarization, under several incidence angles of (a) $0^{\circ}$, (b) $30^{\circ}$, (c) $65^{\circ}$ and (d) $88^{\circ}$. 
frequency mean point of Gap VII moves from $f_{I}=1.162 \mathrm{GHz}$ at $\theta=0^{\circ}$ (a) to $f_{F}=1.163 \mathrm{GHz}$ at $\theta=88^{\circ}(\mathrm{d})$, in the figure. Although, the width of the band-gaps remains invariant, their minimum values decreases more and more by increasing the incidence angle. For example, the minimum value of Gap X decreases from $f_{I}=0.09 \mathrm{GHz}$ at $\theta=0^{\circ}$ (a) to $f_{F}=0 \mathrm{GHz}$ at $\theta=88^{\circ}$ (d). Thus, almost all of the band-gaps reach zero or near-zero transmittance, and lots of narrow transmittance peaks appear if $\theta=88^{\circ}$. The behavior of the band-gaps in Fig. 4 is also the same as what we have in Fig. 3. Nevertheless, the changes are more perceptible here. For example, the frequency mean point of Gap $\Omega$ is $2.45 \mathrm{GHz}$ in Fig. 4(TE)(a), while the value becomes $2.41 \mathrm{GHz}$ in Fig. $4(\mathrm{TE})(\mathrm{d})$. Thus, the amount of change is $0.04 \mathrm{GHz}$ for Gap $\Omega$, while the amount was $0.001 \mathrm{GHz}$ for Gap VII in Fig. 3(TE). In addition, the width the of band-gaps also becomes narrower by decreasing the incidence angle in Fig. 4. For example, the width of Gap $\Omega$ is $0.1 \mathrm{GHz}$ at $\theta=0^{\circ}$, while the value is $0.5 \mathrm{GHz}$ at $\theta=88^{\circ}$. As shown in Fig. 3,4 and 5 , some parts of the band-gaps remain invariant with changing the incidence angle, called omnidirectional band-gaps. These types of band-gaps do not permit EM waves to propagate via the structure at any incidence angle. For instance, one of these band-gaps is colored in Fig. 4(TE), whose width is $\Delta f=0.023 \mathrm{GHz}$. Despite Fig. 3 and 4 , the PBGs shift to higher frequencies by increasing the incidence angle in Fig. 5. Here, the shift value is greater compared to the previous figures, and the mean point of Gap $\rho$ is $4.11 \mathrm{GHz}$ in Fig. 5 (TE)(b), while the value reaches $4.32 \mathrm{GHz}$ in Fig. $5(\mathrm{TE})(\mathrm{d})$. The minimum value of the transmittance gaps is not zero at the lower incidence angles so that the minimum value of Gap $\rho$ is 0.52 in Fig. 5(TE)(a). All of the above analyses could also be expressed for the TM polarization curves of the transmittance.

\subsection{Lattice constants}

In this case, the proposed structure is imposed on a perpendicular incident $\mathrm{GHz}$ beam $\left(\theta=0^{\circ}\right)$ in the frequency range of $1.45 \mathrm{GHz}$ to $2.7 \mathrm{GHz}$ with the total period number of $N=5$ for TE polarization. Here, the transmittance quantity is evaluated with several thicknesses of the constituent layers as $d_{A}=d_{B}=$ (a) $2 \mathrm{~mm}$, (b) $3 \mathrm{~mm}$, (c) $4 \mathrm{~mm}$ and (d) $5 \mathrm{~mm}$. As shown in Fig. 6, the increase in lattice constants causes the number of PBGs to be increased. Generally, there is a lower number of band-gaps with a broader width for the smaller lattice constants, while we have more band-gaps with a lower width by increasing the lattice constants. For example, there are three band-gaps in Fig. 6(a), whose width average is $0.138 \mathrm{GHz}$. On the other hand, there are six band-gaps in Fig. 6(d) with the mean width of $0.843 \mathrm{GHz}$.

\subsection{Period number}

The transmittance spectrum of the structure is shown in Fig. 7 for several applied total period number of $N=$ (a) 2, (b) 4, (c) 6 and (d) 8 with TE polarization. Although, the width of Gaps XIII, XIV and $\eta$ decreases by increase in the period number, the width of the other band-gaps remains invariant. By increasing 


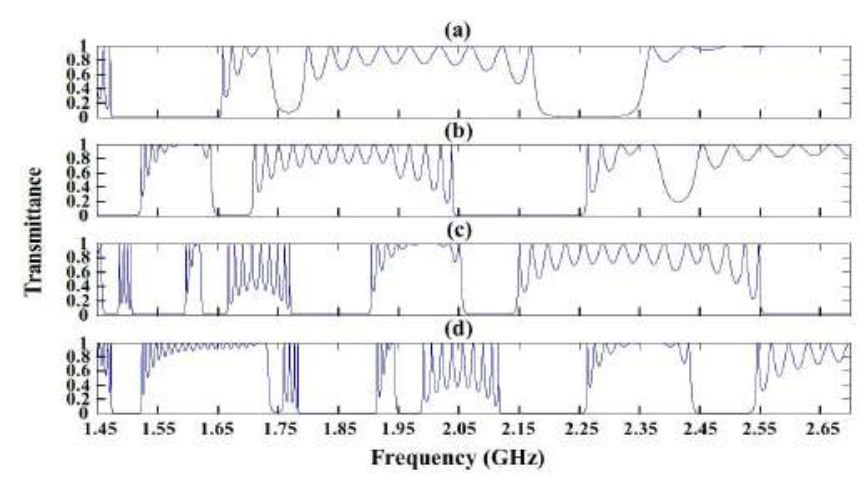

Fig. 6 The transmittance spectrum of the normal incident wave $\left(\theta=0^{\circ}\right)$ in the frequency range of $1.45 \mathrm{GHz}$ to $2.7 \mathrm{GHz}$ with the total period number $N=5$ under several lattice constants of $d_{A}=d_{B}=$ (a) $2 \mathrm{~mm}$, (b) $3 \mathrm{~mm}$, (c) $4 \mathrm{~mm}$ and (d) $5 \mathrm{~mm}$ for TE polarization.

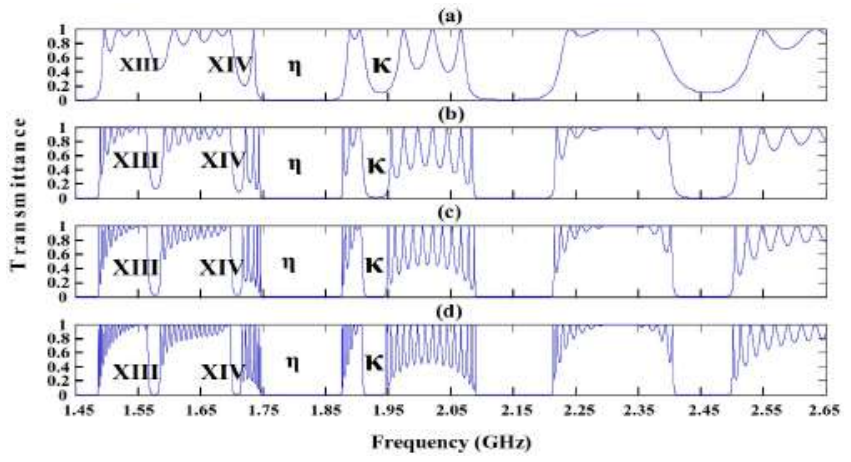

Fig. 7 The transmittance spectrum of the normal incident wave $\left(\theta=0^{\circ}\right)$ in the frequency range of $1.45 \mathrm{GHz}$ to $2.65 \mathrm{GHz}$ with the lattice constants $d_{A}=4 \mathrm{~mm}$ and $d_{B}=5 \mathrm{~mm}$ under several total period numbers of $N=$ (a) 2, (b) 4, (c) 6 and (d) 8 for TE polarization.

the period number, the rate of transmittance changes or transmittance oscillation increases, and the corners of the band-gaps become sharper. The minimum transmittance values of Gaps XIII, XIV and $\kappa$ also reach zero, as the total period number sufficiently increases.

The three-dimensional plot of the transmittance of the proposed structure is demonstrated in terms of frequency and incidence angle in Fig. 8. The left-hand side of the figures (from $\theta=90^{\circ}$ to $0^{\circ}$ ) shows the TM-transmittance, and the right-hand side of the figures (from $\theta=0^{\circ}$ to $90^{\circ}$ ) shows the TE-transmittance. These plots are viewed in line with the transmittance values axis, under several period numbers. As demonstrated in these figures, the band-gaps and the peaks are too narrow and sharp for $0 \mathrm{GHz}$ to $\sim 3 \mathrm{GHz}$, and the number of peaks and the band-gaps are more than the peaks and band-gaps in other investigated frequency ranges. Additionally, there is almost no shift for the lower and upper frequency limits of the PBGs for both TM and TE modes, from $\theta=0^{\circ}$ to $90^{\circ}$, in the frequency range of $0 \mathrm{GHz}$ to $\sim 3 \mathrm{GHz}$. In other words, these band-gaps are completely omnidirectional. On the other hand, the PBGs are broader at the frequencies over 

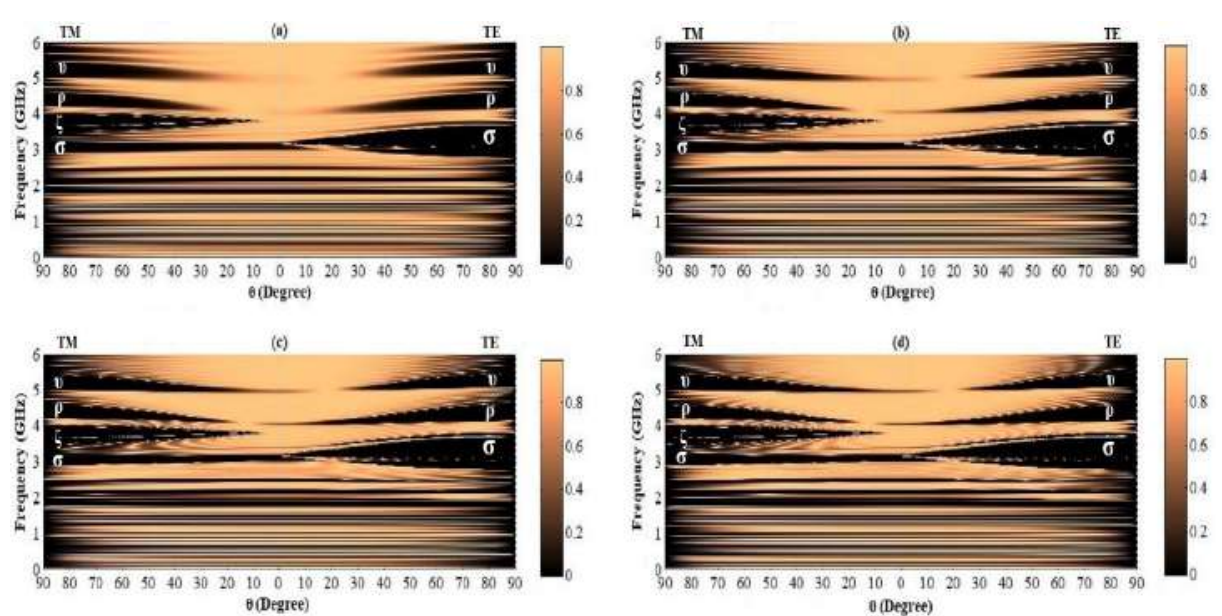

Fig. 8 The three-dimensional projected plots of the transmittance spectrum of the proposed structure are given in terms of frequency and incidence angle under several period numbers as $N=$ (a) 2, (b) 4, (c) 6 and (d) 8 with the lattice constants $d_{A}=4 \mathrm{~mm}$ and $d_{B}=5 \mathrm{~mm}$ for $\mathrm{TE}$ and TM polarizations.

$\sim 3 \mathrm{GHz}$, and their width is not completely invariant. The lower frequency limits of the PBGs are almost constant, and their upper frequency limits shift towards higher frequencies as the incidence angle increases. Therefore, it could be clearly stated that these types of PBGs are partially omnidirectional, and their omnidirectional width is almost equal to their width at lower degrees of the incidence angle. As it is expected from Fig. 7, the number of band-gaps and peaks becomes greater with increase in the period number, while the main band-gaps like $\sigma, \zeta, \rho$ and $\nu$ remain invariant. For instance, see the transmittance region between Gaps $\rho$ and $\nu$ in Fig. 8(a) with four peaks and three sub-band-gaps. As shown in Fig. 8(b), (c) and (d), the number of peaks and band-gaps becomes greater and their width becomes narrower, while the width of the region almost remains invariant.

\subsection{Metamaterial-dielectric composition}

This section deals with the deposition of dielectric layer instead of one type of the utilized metamaterials in the structure. In other words, the period of the structure will be constructed by dielectric and metamaterial layers. First of all, we substitute metamaterial $B$ with a dielectric layer, including different permittivity values. Thus, we have a type of metamaterial layer as $A$, and dielectric layer as $B$, to which several permittivities are allocated, to evaluate the tunability of the obtained PBGs. The applied permittivities of layer $B$ are $\varepsilon_{B L}=1,2,2.5$ and 3 as lower values, and $\varepsilon_{B H}=4.5,5.5,6.5$ and 7.5 as higher values, which their correspondent transmittance respectively demonstrated in Fig. 9(L) and Fig. 9(H). In Fig. $9(\mathrm{~L})$, the structure is evaluated in the frequency range of $1.5 \mathrm{GHz}$ to $3.5 \mathrm{GHz}$, which by increasing the dielectric permittivity value, Gap $\alpha$ moves towards the lower frequencies, and its width also becomes narrower. The width and position (frequency mean point) of this gap are respectively equal to $\Delta f_{\alpha}=0.736 \mathrm{GHz}$ 
and $f_{\alpha}=2.583 \mathrm{GHz}$ at $\varepsilon_{B}=1$ (a), while the values are $\Delta f_{\alpha}=0.138 \mathrm{GHz}$ and $f_{\alpha}=2.224 \mathrm{GHz}$ at $\varepsilon_{B}=3(\mathrm{~d})$. As obviously shown in Fig. $9(\mathrm{~L})(\mathrm{d})$, the minimum value of Gap $\alpha$ is not zero. In other words, there is no complete PBG at this permittivity value and frequency range. In this frequency range, there is a lack of PBG from $\varepsilon_{B}=2.6$ to 4.7. Nevertheless, the behavior of the band-gaps, which are relevant to the higher permittivity values, is distinct from what we have at the lower values in accordance with Fig. 9(H). The applied permittivities corresponding to Fig. $9(\mathrm{H})$ are $\varepsilon_{B H}=4.5$ (a), 5.5 (b), 6.5 (c) and 7.5 (d). Here, the applied frequency range for the incident beam is $1.25 \mathrm{GHz}$ to $3 \mathrm{GHz}$. As shown in the figure, Gap $\beta$ becomes broader as much as $0.214 \mathrm{GHz}$, and moves towards the lower frequencies as much as $0.158 \mathrm{GHz}$, by increasing the permittivity.
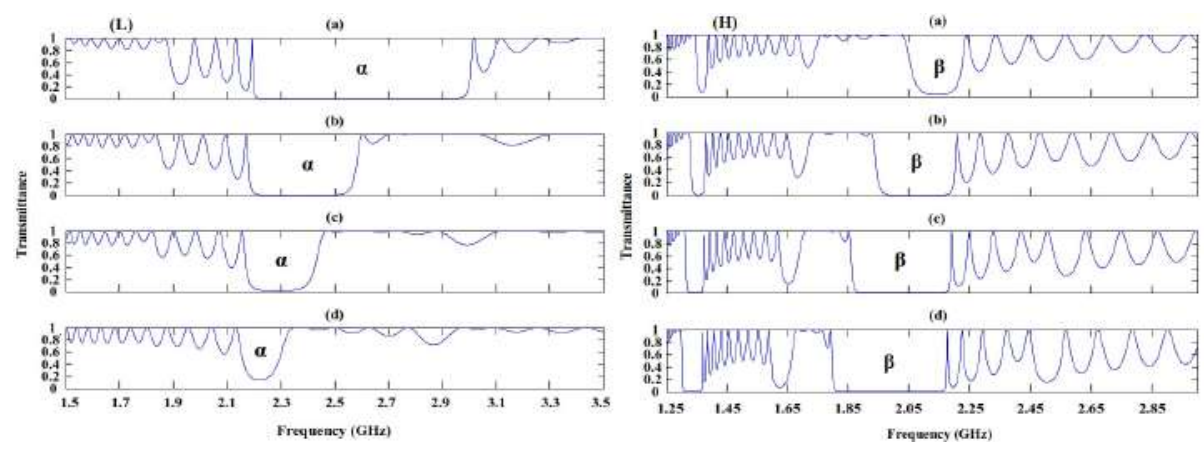

Fig. 9 The transmittance spectrum of the proposed structure is evaluated for several permittivity values of (L) $\varepsilon_{B}=$ (a) 1 , (b) 2 , (c) 2.5 and (d) 3 , as lower values, and (H) $\varepsilon_{B}=(\mathrm{a}) 4.5$, (b) 5.5 , (c) 6.5 and (d) 7.5, as higher values, for TE polarization, where metamaterial layer $\mathrm{A}$ is preserved and metamaterial layer $\mathrm{B}$ is substituted with a dielectric-type layer. Here, the incidence angle is $\theta=0^{\circ}$, the total period number is equal to $N=2$, and the lattice constants are $d_{A}=4 \mathrm{~mm}$ and $d_{B}=5 \mathrm{~mm}$.
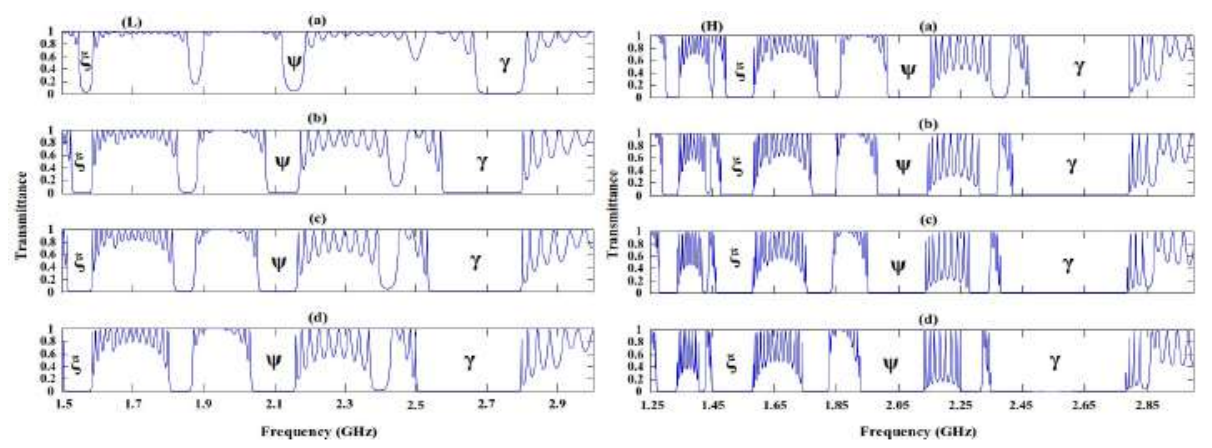

Fig. 10 The transmittance spectrum of the proposed structure is evaluated for several permittivity values of (L) $\varepsilon_{A}=$ (a) 2 , (b) 3 , (c) 3.5 and (d) 4 , as lower values, and (H) $\varepsilon_{A}=($ a) 4.5 , (b) 5.5 , (c) 6.5 and (d) 7.5, as higher values, for TE polarization, where metamaterial layer $\mathrm{B}$ is preserved and metamaterial layer $\mathrm{A}$ is substituted with a dielectric-type layer. Here, the incidence angle is $\theta=0^{\circ}$, the total period number is equal to $N=2$, and the lattice constants are $d_{A}=4 \mathrm{~mm}$ and $d_{B}=5 \mathrm{~mm}$. 
On the other hand, metamaterial $B$ is preserved, and metamaterial $A$ is substituted with a type of dielectric layer. Here, the applied permittivity values for the dielectric layer are $\varepsilon_{A L}=2,3,3.5$ and 4 as lower values. As the structure does not have any PBGs at $\varepsilon_{A}=1$, the value is omitted. Hence, the evaluation starts from $\varepsilon_{A}=2$. Here, the behavior of the PBGs is similar to what we have in Fig. $9(\mathrm{H})$. However, the number of band-gaps is more than the number in Fig. 9(H) in a particular range of frequency. In this section, the common phenomenon among all of the diagrams is that the increase in dielectric permittivity turns the uncomplete band-gaps into the complete ones. The applied permittivities in Fig. 10(H), $\varepsilon_{A H}=4.5,5.5,6.5$ and 7.5, make the band-gaps broader. Additionally, the changes of transmittance are intensified out of the PBGs by increasing the permittivity. For example, the width of Gap $\gamma$ is $\Delta f_{\gamma}=0.294 \mathrm{GHz}$ in Fig. $10(\mathrm{~L})(\mathrm{d})$, while the value is $\Delta f_{\gamma}=0.435 \mathrm{GHz}$ in Fig. $10(\mathrm{H})(\mathrm{d})$. It could be understood from Fig. 9 and 10 (except Fig. 9(L)) that the lower frequency limits of the most PBGs move faster than the upper limits, thus the changes of the upper limits could be ignored. For instance, see Gaps $\xi$ and $\Psi$ etc. It should be noted that a similar analysis could be represented for the other frequency ranges surrounding the studied ranges. The TM polarization curves relevant to this section are also the same as what are demonstrated for the TE polarization.

\section{Conclusion}

Given that the arrangement of the PC constituent elements is a major determinant in their outcomes, utilizing the QP arrays could be a beneficial option for changing the arrangement of PC structures. The proposed quasi-PC, that contains two types of DNG metamaterial with T-M sequence, eventuates some narrow and wide omnidirectional PBGs such as band-gaps $\kappa$ and $\eta$, respectively, in Fig. 4. In accordance with Fig. 3(d), 4(d) and 5(d), the sharp transmittance peaks and the narrow PBGs appear for both TE and TM incident polarized beam, as the incidence angle increases. It is understood from Fig. 8 that there are lots of desirable narrow band-gaps and of course sharp peaks in the frequency range of $0 \mathrm{GHz}$ to $\sim 2.5 \mathrm{GHz}$. In this frequency range, most of the band-gaps are also omnidirectional for both TE and TM polarization mode. The band-gaps upper than the frequency range are partially omnidirectional. In the current decay, the quasi-PC based DNG metamaterials have been a focal point of a wide range of various fields and applications such as optics, photonics, optoelectronics, communications, imaging, sensing as well as reflectors, polarization filters, splitters [6], etc. The proposed structure could play a significant role in promoting and enhancing the quality and efficiency of the above-mentioned fields.

\section{Funding}

No funds, grants, or other support was received. 


\section{Conflicts of interest}

The authors have no conflicts of interest to declare that are relevant to the content of this article.

\section{Data availability}

All data generated or analysed during this study are included in this published article.

\section{References}

1. V.G. Veselago, Physics-Uspekhi 10(4), 509 (1968)

2. M.A. Noginov, V.A. Podolskiy, Tutorials in metamaterials (CRC press, 2011)

3. O. Gunduz, C. Sabah, Journal of Computational Electronics 15(1), 228 (2016)

4. H.I. Lin, K.C. Shen, S.Y. Lin, G. Haider, Y.H. Li, S.W. Chang, Y.F. Chen, Scientific reports $8(1), 1$ (2018)

5. F. Segovia-Chaves, H. Vinck-Posada, Physica B: Condensed Matter 543, 7 (2018)

6. O. Soltani, J. Zaghdoudi, M. Kanzari, Photonics and Nanostructures-Fundamentals and Applications 38, 100744 (2020)

7. T.W. Sheu, R. Chang, Y. Chang, J. Li, Journal of Computational Electronics 13(1), 313 (2014)

8. D. Yan, J. Li, L. Jin, Laser Physics 29(2), 025401 (2018)

9. A. Baseri, A. Keshavarz, A. Hatef, Journal of Applied Physics 127(21), 214304 (2020)

10. P. King, Acoustic properties of periodically and quasi-periodically modulated waveguides. Ph.D. thesis, M. Sc. Thesis, The University of Salford (2007)

11. M. Maksimović, Z. Jakšić, Journal of Optics A: Pure and Applied Optics 8(3), 355 (2006)

12. R. Merlin, K. Bajema, R. Clarke, F.Y. Juang, P. Bhattacharya, Physical review letters $\mathbf{5 5}(17), 1768(1985)$

13. C. Xu, X. Xu, D. Han, X. Liu, C.P. Liu, C.J. Wu, Optics communications 280(1), 221 (2007)

14. W. Liang, S. He, L. Chen, Optics Express 11(11), 1283 (2003)

15. I.V. Shadrivov, A.A. Sukhorukov, Y.S. Kivshar, Physical review letters 95(19), 193903 (2005)

16. A.H. Aly, W. Sabra, Journal of Superconductivity and Novel Magnetism 29(8), 1981 (2016)

17. Z. Luo, M. Chen, J. Deng, Y. Chen, J. Liu, Optik 127(1), 259 (2016)

18. C.R. Lee, S.H. Lin, S.M. Wang, J.D. Lin, Y.S. Chen, M.C. Hsu, J.K. Liu, T.S. Mo, C.Y. Huang, Journal of Materials Chemistry C 6(18), 4959 (2018)

19. Q. Wang, X. Wang, L. Zhang, Y. Wang, W. Qiao, X. Han, X. Cai, W. Yu, Applied optics $\mathbf{5 8}(1), 94(2019)$

20. G. Palai, A. Nayyar, R. Manikandan, B. Singh, Optik 179, 740 (2019)

21. E. Liviotti, Journal of Physics: Condensed Matter 8(27), 5007 (1996)

22. M. Vasconcelos, E. Albuquerque, A. Mariz, Journal of Physics: Condensed Matter 10(26), 5839 (1998)

23. X.H. Deng, W.Q. Hong, H. Ouyang, et al., Physics Procedia 22, 360 (2011)

24. F. Xi, L. Hu, The European Physical Journal D 66(2), 1 (2012)

25. M. Born, E. Wolf, Principles of optics: electromagnetic theory of propagation, interference and diffraction of light (Elsevier, 2013)

26. W. Li-Gang, L. Nian-Hua, L. Qiang, Z. Shi-Yao, Physical Review E 70(1), 016601 (2004)

27. S.K. Awasthi, A. Mishra, U. Malaviya, S. Ojha, Solid State Communications 149(33-34), 1379 (2009)

28. L. Moretti, I. Rea, L. Rotiroti, I. Rendina, G. Abbate, A. Marino, L. De Stefano, Optics Express 14(13), 6264 (2006) 
Figures
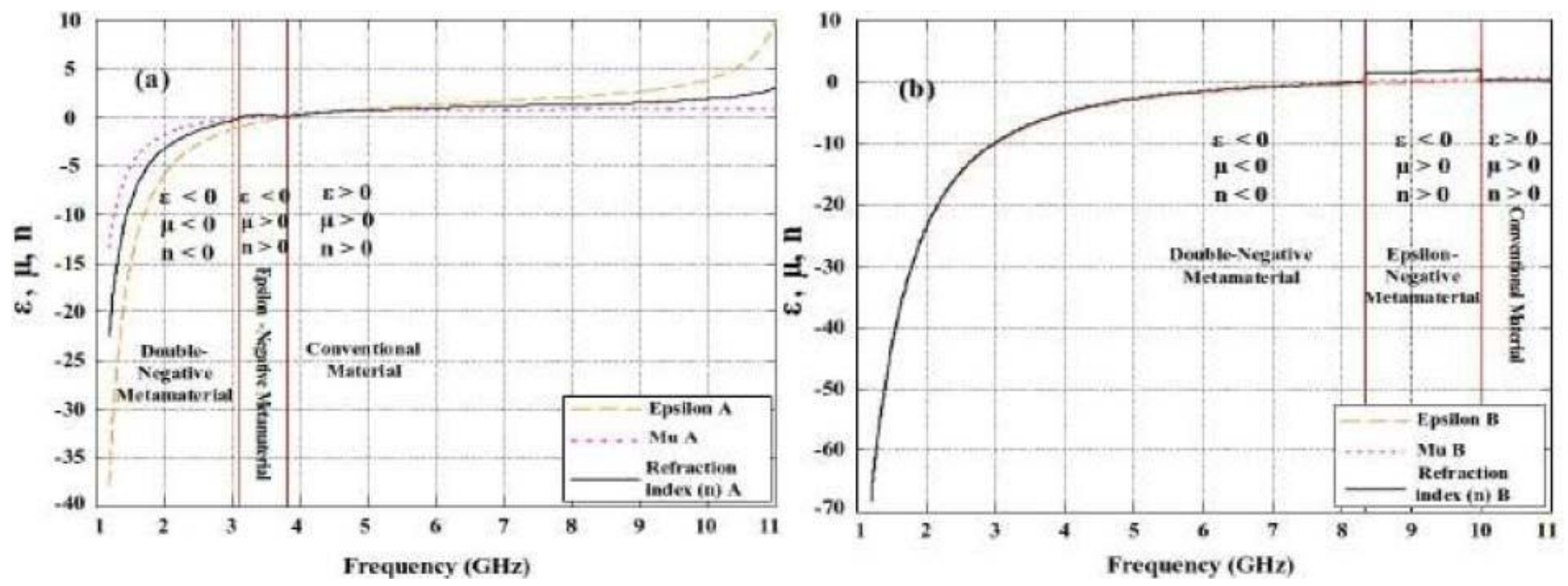

Figure 1

The function of permittivity, permeability and refraction index of metamaterials (a) A and (b) B in terms of frequency. According to the diagrams, type of the metamaterials changes across different frequency ranges.

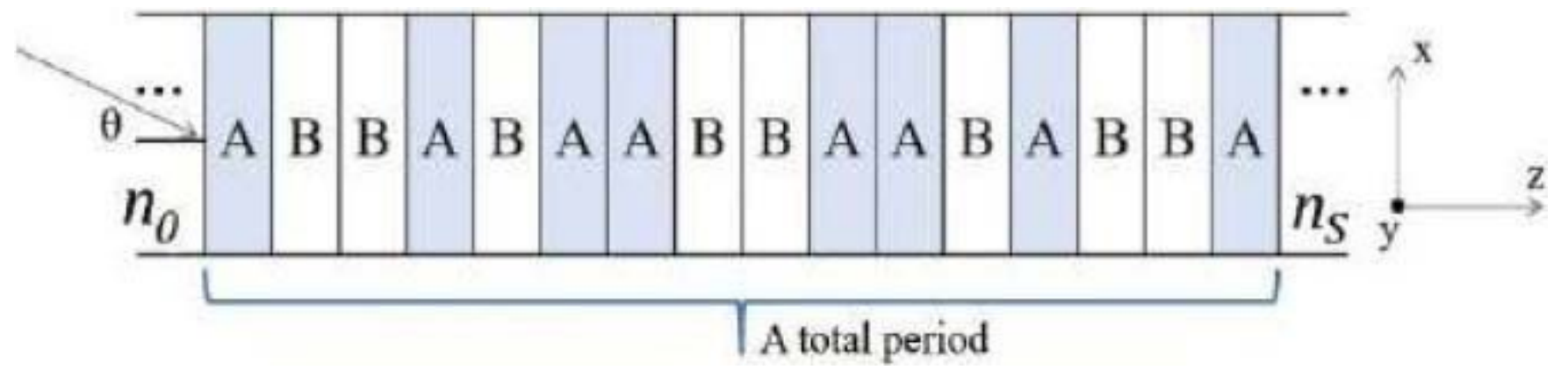

Figure 2

"Please see the Manuscript PDF file for the complete figure caption". 

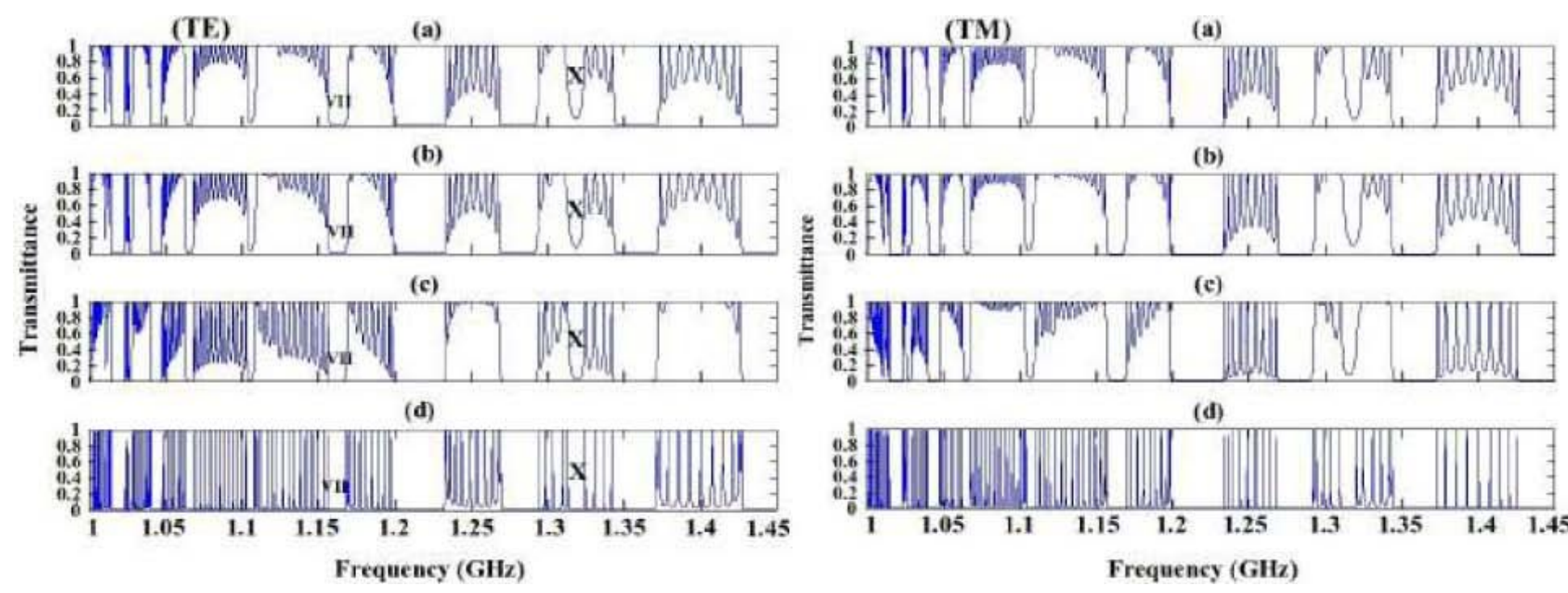

(d)

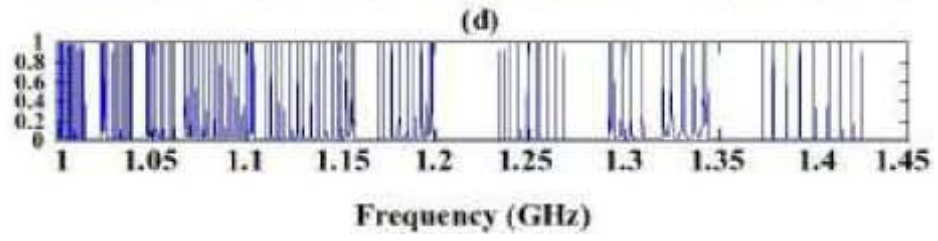

Figure 3

The transmittance spectrum of the incident wave in the frequency range of $1 \mathrm{GHz}$ to $1.45 \mathrm{GHz}$, to a quasi$\mathrm{PC}$, which contains two types of DNG metamaterial with the fourth-order of the T-M arrangement for the TE and TM polarization under several incidence angles of (a) $0^{\circ}$, (b) $30^{\circ}$, (c) $65^{\circ}$ and (d) $88^{\circ}$.
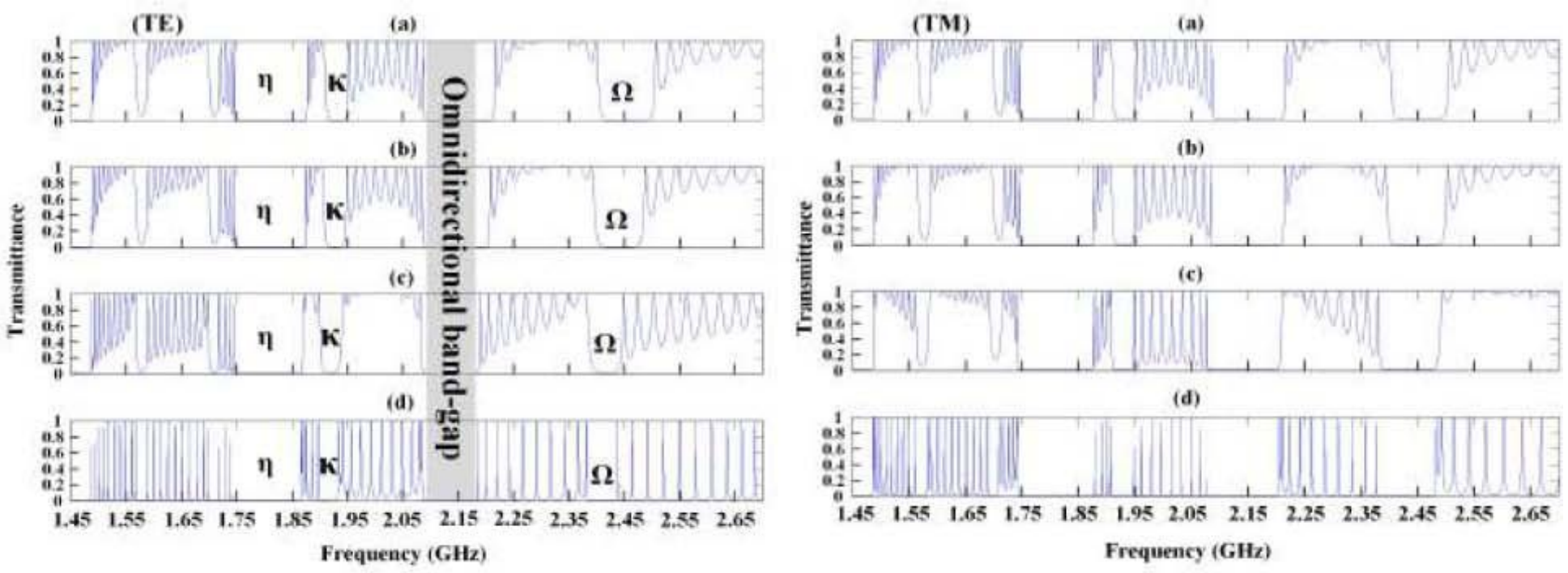

Figure 4

The transmittance spectrum of the incident wave in the frequency range of $1.45 \mathrm{GHz}$ to $2.7 \mathrm{GHz}$, to a quasi-PC, which contains two types of DNG metamaterial with the fourth-order of the T-M arrangement for the TE and TM polarization under several incidence angles of (a) $0^{\circ}$, (b) $30^{\circ}$, (c) $65^{\circ}$ and (d) $88^{\circ}$. 

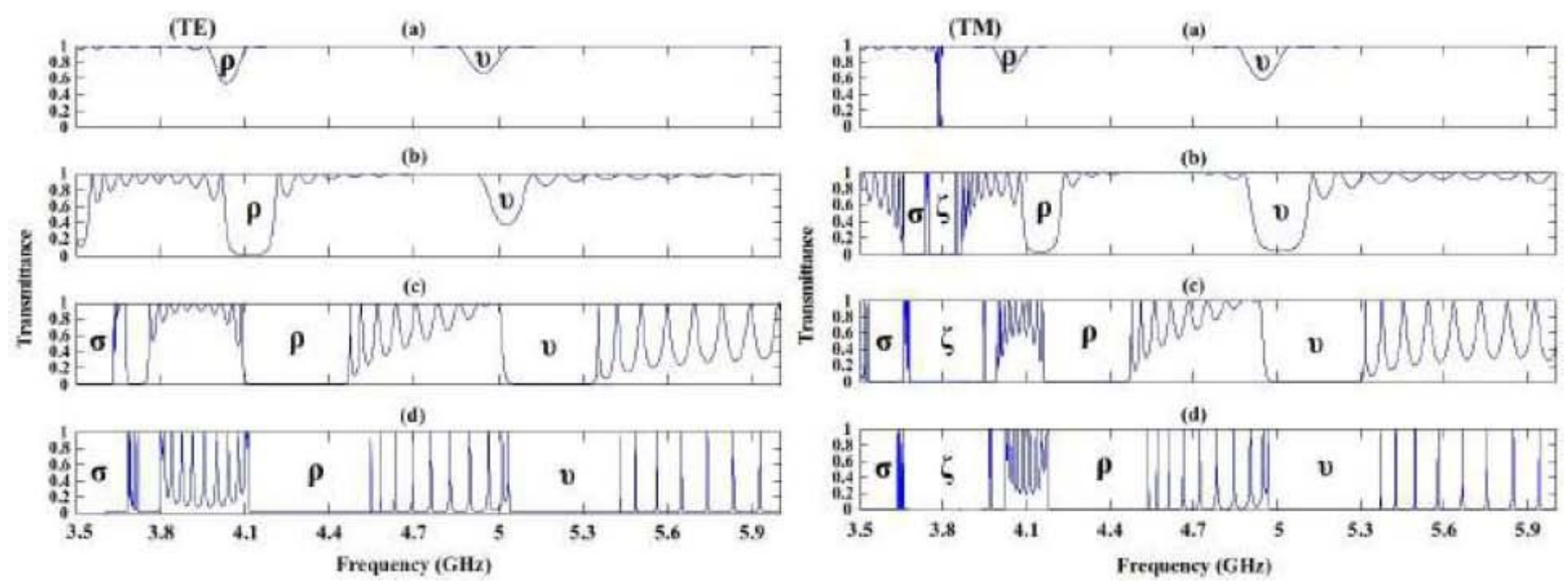

Figure 5

The transmittance spectrum of the incident wave in the frequency range of $3.5 \mathrm{GHz}$ to $6.0 \mathrm{GHz}$, to a quasi-PC, which contains two types of DNG metamaterial with the fourth-order of the T-M arrangement for the TE and TM polarization, under several incidence angles of (a) $0^{\circ}$, (b) $30^{\circ}$, (c) $65^{\circ}$ and (d) $88^{\circ}$.

(a)
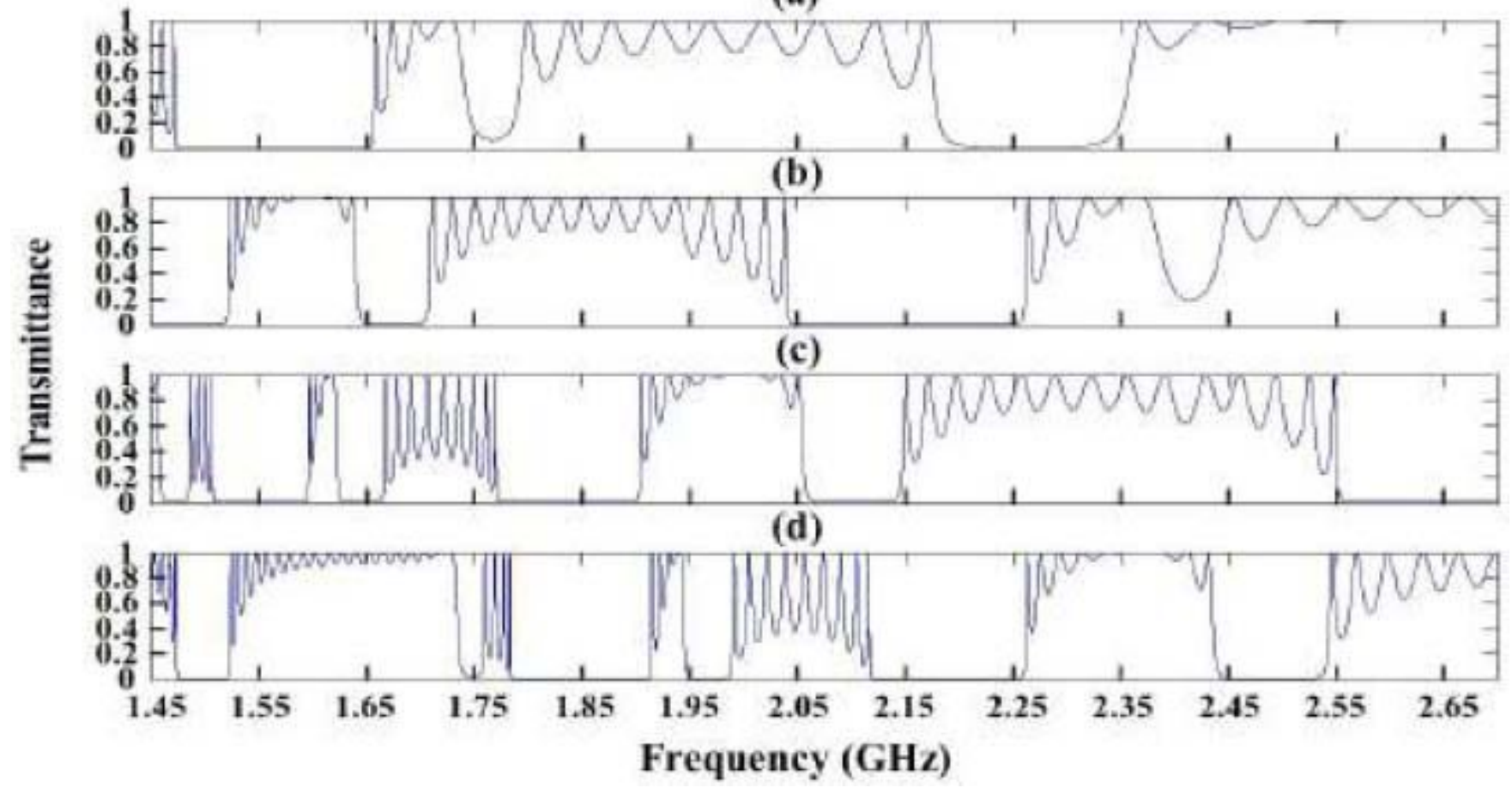

Figure 6

"Please see the Manuscript PDF file for the complete figure caption". 
(a)

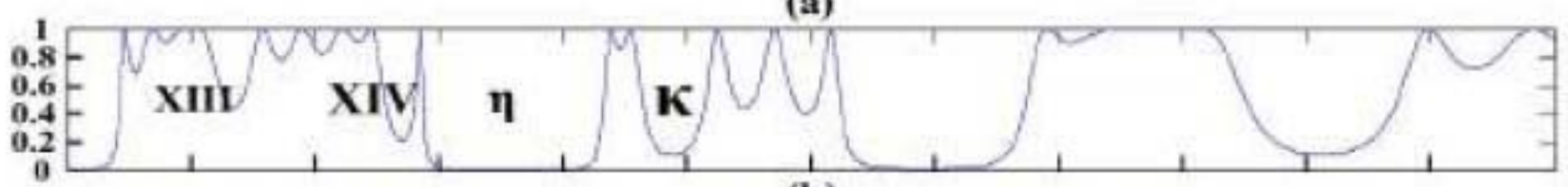

(b)

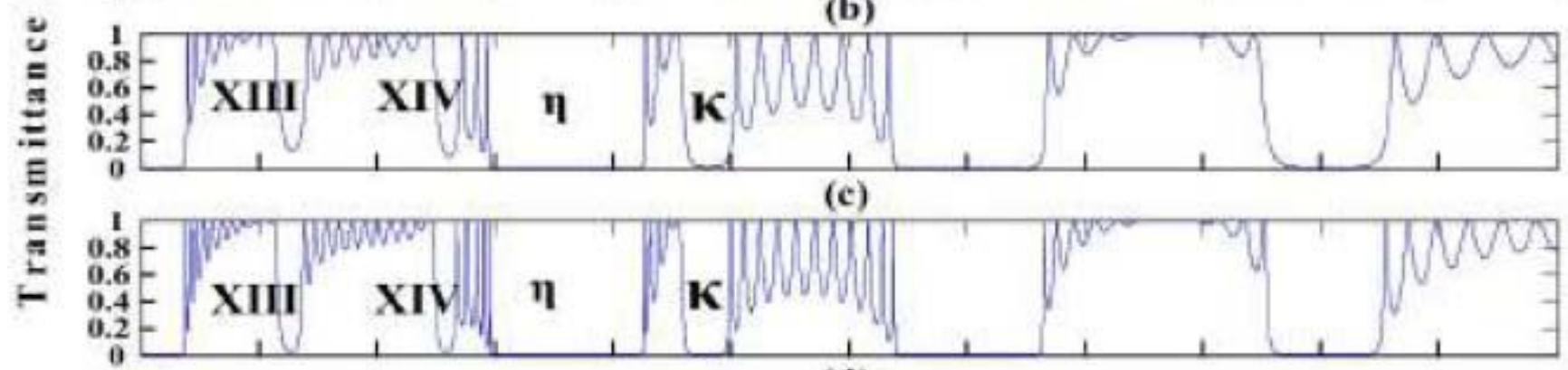

(d)

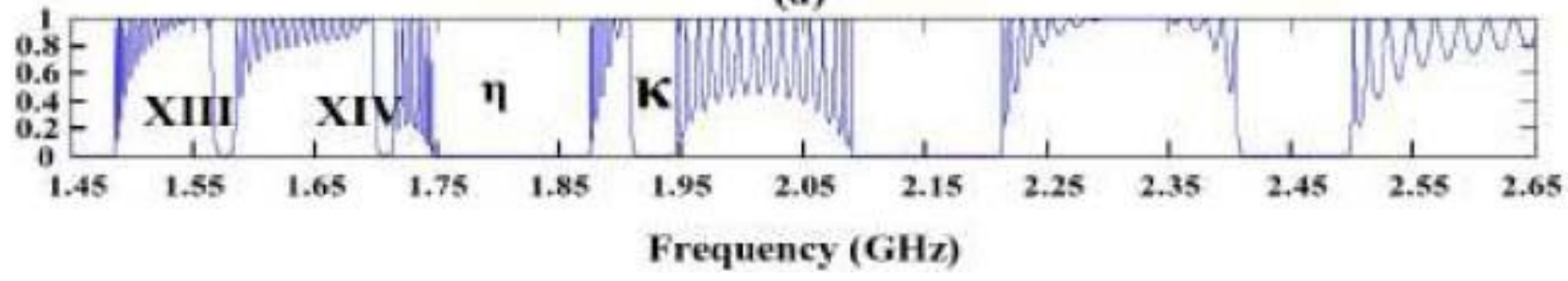

Figure 7

"Please see the Manuscript PDF file for the complete figure caption".
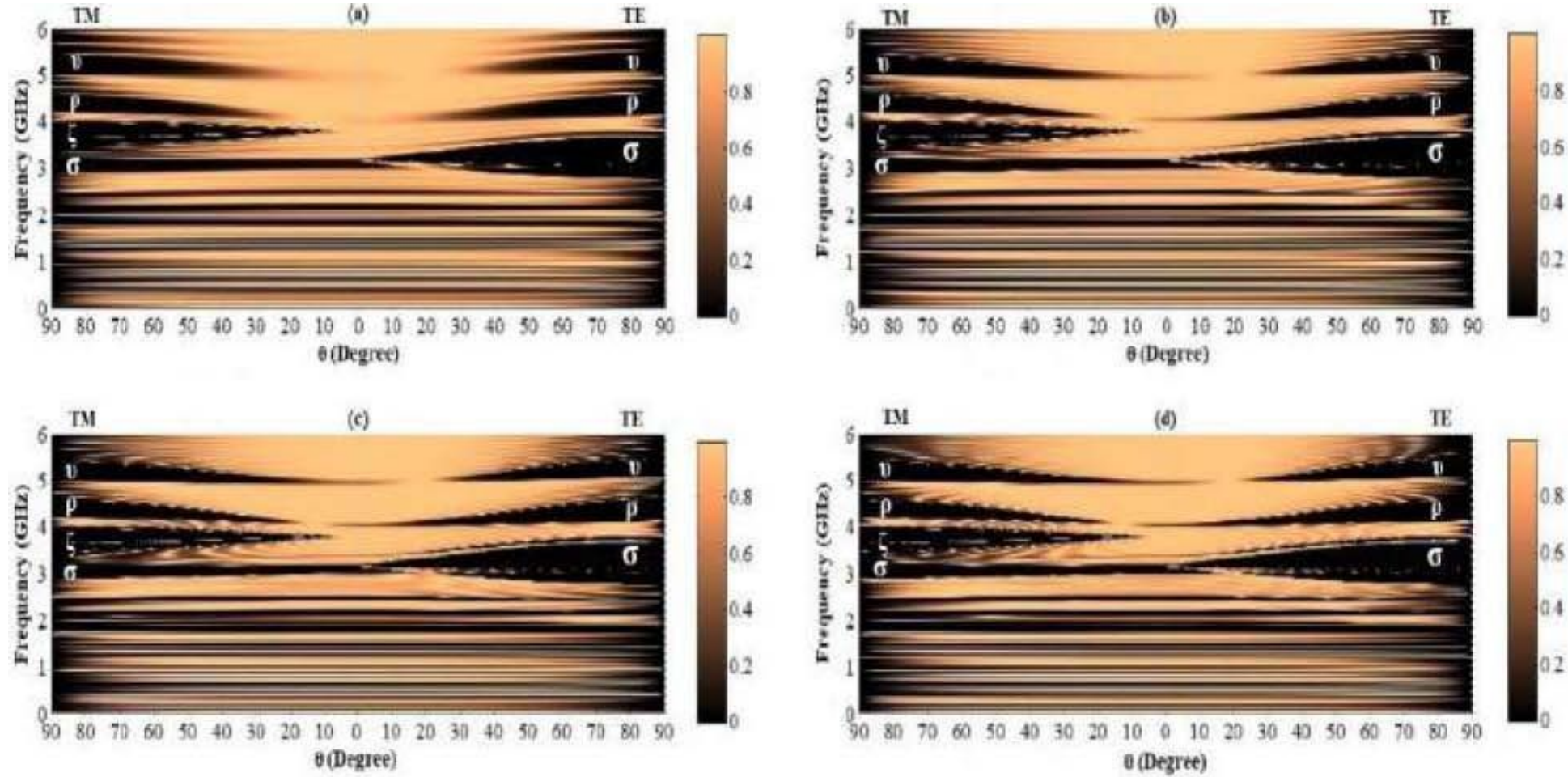

Figure 8

"Please see the Manuscript PDF file for the complete figure caption". 
(L)
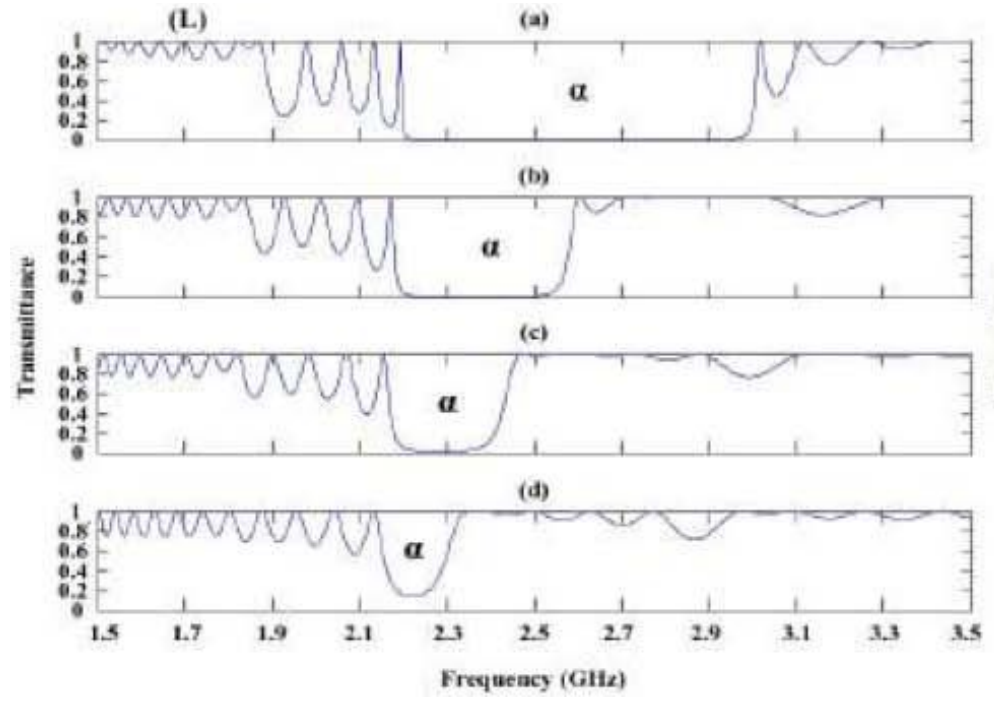
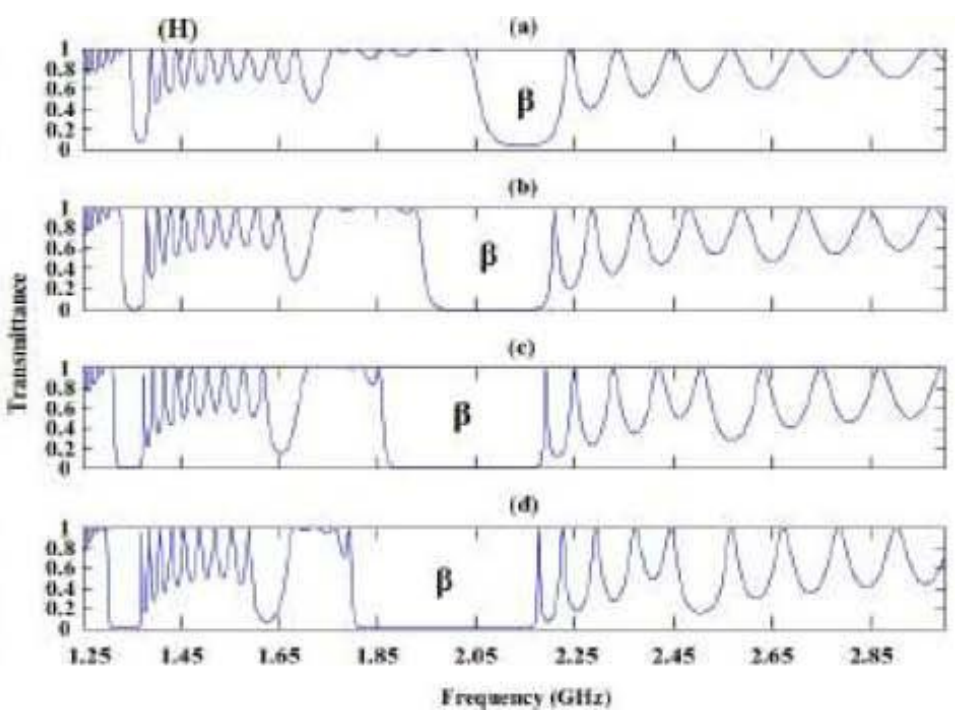

\section{Figure 9}

"Please see the Manuscript PDF file for the complete figure caption".
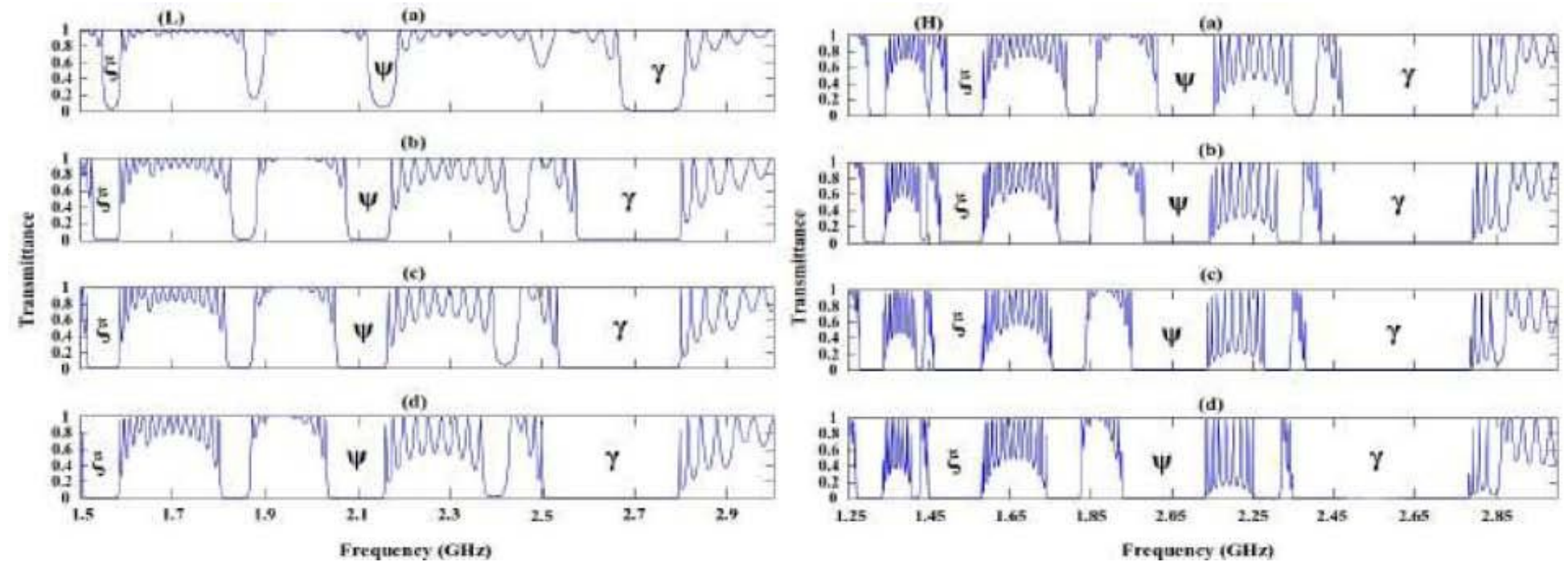

Figure 10

"Please see the Manuscript PDF file for the complete figure caption". 